\title{
Are presentation frequency and spatial numerosity distinct attributes of memory?
}

\author{
DOUGLAS L. HINTZMAN \\ University of Oregon, Eugene, Oregon 97403
}

\begin{abstract}
Twenty-five words were assigned to all combinations of the spatial numerosities $1-5$ and the presentation frequencies 1-5. Frames displaying the appropriate number of copies of each word were repeated the appropriate number of times, and subjects attempted to memorize the words' numerosities. A combination frequency-judgment and numerosity-recall test was then given. Numerosity showed no tendency to intrude in the frequency-judgment task, and there was only marginal evidence for interference in the opposite direction. The results are interpreted as further support for the view that presentation frequency has a privileged representation, which is not shared with other kinds of quantitative information in memory.
\end{abstract}

A fundamental assumption of multiple-trace theories is that presentation frequency has a unique or privileged status as an attribute of memory (Hintzman, 1976; Hintzman, Nozawa, \& Irmscher, 1982). That is, the number of memory traces of an item provides an analogue representation of the item's frequency, a representation not shared with any other remembered quantity.

To test this assumption, Hintzman et al. (1982) had subjects learn associations between pictures and the digits $1-5$, presenting the pairs from one to five times each. Half the subjects were then shown the pictures and asked to recall the digits. The other half unexpectedly were asked first to judge the presentation frequencies of the pictures, and only then were they given the digit recall test. In the frequency-judgment task, subjects showed no tendency to mistakenly give the digit associates, and only the second group of subjects (those who had judged the frequencies of the pictures first) showed a tendency to intrude frequency information in digit recall.

These results were interpreted as follows: Incidentally acquired frequency information is not represented propositionally; rather, it is retrieved from a privileged "format," as multiple-trace theory assumes. As such, it is not confused with quantitative information learned propositionally or associatively, such as that linking the members of the picture-digit pairs. However, on a frequency-judgment test, the frequency information becomes propositionalized (i.e., copied from its privileged representation into a general, propositional form) and, so, becomes capable of subsequently interfering with quantitative information that has been associatively learned.

This material is based upon work supported by the National Science Foundation under Grant BNS-7824987. Requests for reprints should be sent to Douglas L. Hintzman, Department of Psychology, University of Oregon, Eugene, Oregon 97403.
In the present study, the question of interest was whether similar mutual noninterference would be displayed by two different kinds of frequency information: spatial frequency and temporal, or presentation, frequency. (For simplicity, we will refer to the former as "numerosity" and to the latter as "frequency.") This experiment provides a further, and more stringent, test of the independence prediction. Presumably, if all information were represented propositionally, propositions concerning frequency and numerosity would be similar in content and likely to be confused at retrieval. However, since multiple-trace theory assumes that the underlying representations of frequency and numerosity are qualitatively distinct, it predicts noninterference. An attempt to retrieve and evaluate one kind of information should not mistakenly retrieve the other.

The basic design of the experimental task was similar to that of Experiment 1 of Hintzman et al. (1982). Twenty-five words were assigned at random to all combinations of the frequencies $1-5$ and the numerosities 1-5. Frequency was operationalized as the number of presentation frames containing the word, and numerosity was operationalized as the number of copies of the word per frame. After studying the sequence of frames under instructions only to learn the numerosities of particular words, subjects were given a test on which, for each word, they made separate judgments of frequency and numerosity.

\section{METHOD}

\section{Subjects}

A total of 77 University of Oregon students participated for extra credit in undergraduate psychology classes. They were tested in small groups of varying size. The subjects had served in a previous experiment in which they judged the relative frequencies of pairs of pictures, receiving two different tests 2 weeks apart. The present experiment was conducted in the same session as, and immediately after, the second such test. 


\section{Design and Materials}

A pool of 25 common three-letter English nouns was used. Eight different lists were generated by computer. For each, the words were randomly assigned to the cells of a 5 (frequency = $1-5$ ) by 5 (numerosity $=1-5$ ) matrix. The eight lists, each consisting of 75 computer-generated frames, were recorded on a video cassette recorder for presentation on a $25 \times 20 \mathrm{~cm}$ television screen. For each numerosity value, the appropriate number of copies of the word were arranged spatially in a frame as are spots on a die (i.e., possible locations were the center of the screen and points indented $4 \mathrm{~cm}$ diagonally from each of the four corners). For numerosities of 2 and 3, the upper left to lower right diagonal configuration was used. The frequency value determined the number of identical frames that were generated. All such frames were then ordered randomly, with the restriction that two frames of the same word could not occur in succession. The presentation rate was $2.5 \mathrm{sec} /$ frame (2-sec exposure and .5 -sec blank). Between 7 and 12 subjects were shown each list.

Each group of subjects (defined by the eight lists) had a different test form, on which the 25 words were listed in random order. To the right of each word was printed "presentations: $\begin{array}{lllll}1 & 2 & 3 & 4 & 5\end{array}$," followed by "words per presentation: 12345 ." Subjects indicated their judgments by circling one digit in either set.

\section{Procedure}

The possible spatial arrangements of words on the screen were described to the subjects, and they were told that their task was to learn the appropriate numerosity of each word. They were further informed that, although words would be repeated, the number of copies per frame associated with a particular word would not change. The list was shown using the video recorder, and then the test forms were distributed. Instructions for the test clearly differentiated between the number of frames or presentations and the number of copies of a word per frame, and subjects were instructed to make separate judgments of each by circling the appropriate responses on the test form. To boost motivation, the purpose of the experiment was conveyed (correctly) as one of determining whether the subjects could avoid confusing the two values.

\section{RESULTS}

In order to give each list equal weight in all analyses, the data from all subjects having the same list were collapsed into one "macrosubject." All statistics to be reported, therefore, are based on $\mathrm{N}=8$.

Mean judged frequencies are shown as a function of numerosity and frequency in Figure 1 . The different frequencies clearly produced mean judgments at different levels. However, the curves show no tendency toward positive slope, which would indicate intrusions of numerosity into judgments of frequency. Overall means for numerosities 1.5 collapsed over frequency were: $2.85,2.72,2.78,2.83$, and 2.92 . A correlational analysis showed that frequency accounted for $92.5 \%$ of the variance among the means in Figure 1 and numerosity accounted for only .4\%.

A planned-comparisons ANOVA tested for a nonlinear effect of frequency using the coefficients -6 , $-2,1,3$, and 4 for frequencies $1-5$, respectively, for a linear effect of numerosity and for the multiplicative interaction of the two variables. The effect of frequency was significant $[F(1,7)=211.36, p<.001]$; but the

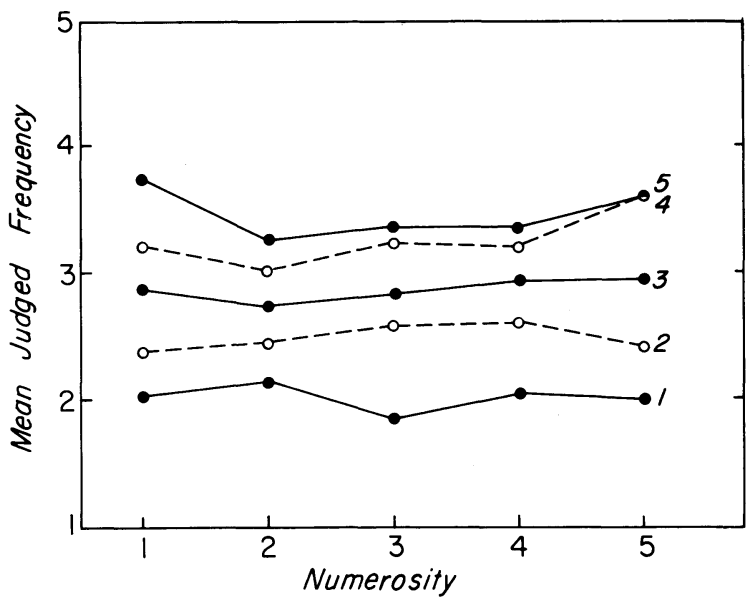

Figure 1. Mean frequency judgment as a function of presentation frequency (the parameter of the curves) and spatial numerosity.

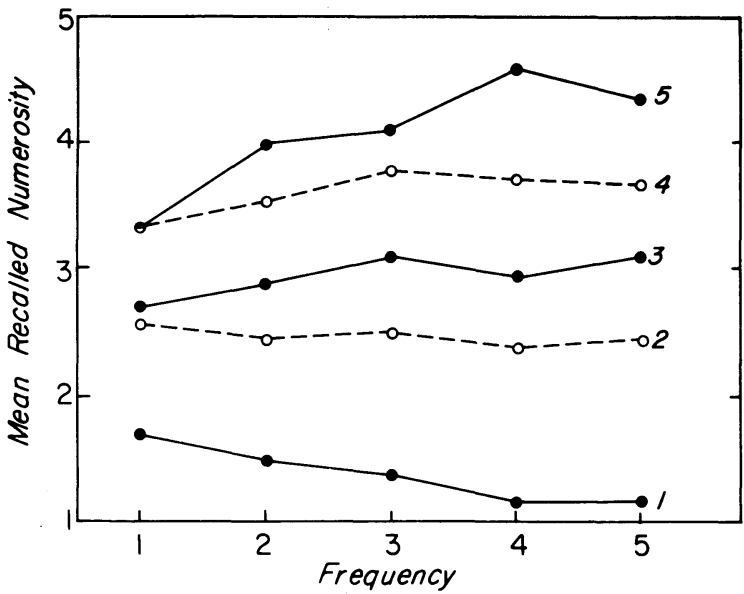

Figure 2. Mean recalled spatial numerosity as a function of numerosity (the parameter of the curves) and presentation frequency.

effect of numerosity and the multiplicative interaction were not $[F(1,7)=.84$ and .74 , respectively $]$. Statistical analyses thus confirm that numerosity had no effect on mean judgments of frequency.

Mean recalled numerosities are presented in Figure 2. Each numerosity value is represented by a different curve. Learning is evidenced by the divergence of the curves as a function of frequency (i.e., each curve approaches its correct numerosity value as an asymptote). Other indications of learning were a decrease over frequencies 1.5 in the standard deviations of the response distributions $(1.05, .92, .88, .74$, and .79 , averaged over numerosities) and an increase in percentage correct $(49 \%, 60 \%, 71 \%, 79 \%$, and $75 \%)$. The question of primary interest is whether recalled numerosity increased with frequency. Means, collapsed over numerosity, suggest that it did somewhat; the overall 
means for frequencies $1-5$ were $2.72,2.87,2.97$, and 2.94. A correlational analysis showed that frequency accounted for $.6 \%$ of the variance among means of Figure 2, and numerosity accounted for $95.8 \%$.

A planned-comparisons ANOVA similar to that done on frequency judgments was performed on recalled numerosity values. The nonlinear effect of frequency was only marginally significant $[F(1,7)=5.37, p<.05$, by a one-tailed test]. The effect of numerosity and the interaction were both highly significant $[F(1,7)=437.5$ and 67.62 , respectively, both ps $<.001]$.

\section{DISCUSSION}

The overall picture emerging from this experiment is that memory for presentation frequency and memory for spatial numerosity are independent to a remarkable degree. The results provide added support to the conclusion of Hintzman et al. (1982) that information regarding presentation frequency is immune to intrusions from other stored information of a quantitative kind. The present support is stronger, however, in two ways: First, probably because words were used instead of pictures, presentation frequency was not remembered as well in the present study as it was in the previous one (compare the present Figure 1 with Figure 1 of Hintzman et al., 1982). It is therefore even less plausible to argue here that associations linking items with frequency values are too well learned to suffer competition from other associations than it was for Experiment 1 of Hintzman et al. (1982).

Second, the intentionally learned values in the present study were spatial frequencies or numerosities, rather than digit associates. The a priori likelihood that propositional encodings of frequency and numerosity would be similar in content and therefore easily confused is higher than it is for frequencies and digit associates. Indeed, if the English language is a guide to the similarity of underlying propositional encodings, one might expect them to be highly confusable. Frequency and numerosity, the terms adopted for short-hand convenience here, are not distinct in their meaning; nor are multiplicity, redundancy, and duplication. To be precise, we have resorted to presentation frequency vs. spatial numerosity, whereas Whitlow and Skaar 1979) distinguished between "frequency of episodes" and "numerosity within an episode." If there is a crisp distinction between the two kinds of frequency at a propositional level, it is not reflected in English vocabulary.

The other aspect of mutual independence, the immunity of recalled numerosity to intrusion by frequency information, is not so clear. The marginally significant main effect of frequency on recalled numerosity could be explained in at least three ways: (1) Subjects made the frequency judgment for each word just before giving the numerosity. If giving a frequency judgment copies the frequency information into the same (propositional) "format" that represents numerosity, then intrusions into attempts to recall numerosity are likely to occur. This is the same explanation given by Hintzman et al. (1982) for a similar main effect of frequency on digit recall, occurring only when the digit-recall test was preceded by judgments of frequency. According to this account, intrusions should occur primarily at the lower frequencies (for which numerosities are poorly learned), and so the expected increase with frequency is curvilinear. The present data have this form. (2) Some subjects may believe that memory improves with spatial numerosity and, therefore, give low numerosities as guesses whenever the test word is one they fail to recognize. This selective-bias hypothesis also predicts the obtained curvilinear function, but it could not plausibly be applied to the digit-recall data of Hintzman et al. (1982). (3) Given the short exposures ( $2 \mathrm{sec})$, subjects may have occasionally, through inattention, failed to notice all copies of a word on its early exposures. This would lead to underestimates of high numerosities at low frequencies, which is roughly the effect that was obtained. The present data do not distinguish among these three explanations, but considering that only the first applies also to the results of Hintzman et al. (1982), it would seem to be the preferable one.

We do not know, of course, that numerosity was propositionally encoded in the present experiment. It is a plausible assumption, since numerosity was learned intentionally and deliberate verbal rehearsal would, presumably, be likely to generate a propositional code. But there may also be an analogue representation of numerosity, as multiple-trace theories assume there is for frequency. A spatial image would be a plausible candidate for such a representation. If so, then the recall of numerosity may have been mediated by both this analogue representation and a propositional code. If our subjects did make use of an analogue representation of numerosity, however, it appears to have been different from that for frequency. The present frequency-judgment results argue strongly for the conclusion (also suggested by Whitlow \& Skaar, 1979) that the underlying representations of spatial numerosity and presentation frequency are distinct.

\section{REFERENCES}

Hintzman, D. L. Repetition and memory. In G. H. Bower (Ed.), The psychology of learning and motivation (Vol. 10). New York: Academic Press, 1976.

Hintzman, D. L., Nozawa, G., \& Irmscher, M. Frequency as a nonpropositional attribute of memory. Journal of Verbal Learning and Verbal Behavior, 1982, 21, 127-141.

Whitlow, J. W., Jr., \& SkaAR, E. The role of numerosity in judgments of overall frequency. Journal of Experimental Psy. chology: Human Learning and Memory, 1979, 5, 409-421.

(Received for publication August 8, 1982.) 